\title{
Immune System Part
}

National Cancer Institute

\section{Source}

National Cancer Institute. Immune System Part. NCI Thesaurus. Code C13033.

Any component of the complex group of organs including the thymus, spleen, tonsils, lymphatic system, hematopoietic system and cells like lymphocytes, granulocytes, monocytes and macrophages that defends the body against infection or disease. 